\title{
is Research Square \\ CCR2 Expression Promotes Diffuse Large B-Cell Lymphoma Survival and Invasion
}

\section{Quan-Quan Hu}

Anhui Medical University https://orcid.org/0000-0002-2352-5390

\section{Zhao-Feng Wen}

Anhui Medical University

\section{Qi-Tang Huang}

Anhui Medical University

\section{Qian Li}

Second Affiliated Hospital of Anhui Medical University

\section{Zhi-Min Zhai}

Second Affiliated Hospital of Anhui Medical University

Yan-Li Li ( $\square$ liyanli_rainbow126@163.com )

Anhui Medical University https://orcid.org/0000-0002-9303-0473

\section{Research Article}

Keywords: Diffuse large B cell lymphoma, CCR2, cell proliferation, cell migration, prognostic marker

Posted Date: February 21st, 2022

DOI: https://doi.org/10.21203/rs.3.rs-1360177/v1

License: (c) (i) This work is licensed under a Creative Commons Attribution 4.0 International License.

Read Full License 


\section{Abstract}

Background CC chemokine receptor 2 (CCR2), has been regarded to involve in tumor growth, angiogenesis, epithelial mesenchymal transition, metastasis and immune escape etc. in recent years, but the role and mechanism in DLBCL has not been reported yet.

Methods We examined the expression of CCR2 and assessed the relationship between the expression level of this receptor and the clinicopathological characteristics of DLBCL patients. Next, characterization of CCR2 expression in the development of DLBCL cell lines was explored by blocking CCR2 expression through a novel antagonist in vitro and in vivo.

Results We showed that CCR2 expression was an independent prognostic marker that influenced DLBCL patients and predicted shorter overall survival (OS) and progression-free survival (PFS). The results showed that CCR2 antagonists inhibited the ability of DLBCL cells to proliferate, migrate and promoted apoptosis by activating the PI3K/Akt signaling pathway and the p38MARK signaling pathway. Furthermore, in vivo experiments, also confirmed that CCR2 antagonists inhibited the proliferation and metastasis of DLBCL neoplasms.

Conclusion Our study demonstrates that CCR2 expression plays an important role in the development of DLBCL by stimulating cell proliferation, migration and anti-apoptosis. The inhibition of CCR2 may, therefore, be a potential target for anticancer therapy in DLBCL.

\section{Introduction}

Diffuse large B cell lymphoma (DLBCL) is the most common subgroup of non-Hodgkin lymphoma in worldwide. It is a phenotypically and genetically heterogeneous disease, accounting for approximately $30-40 \%$ of all cases $[1,2]$. The addition of rituximab to standard CHOP (cyclophosphamide, doxorubicin, vincristine and prednisone) chemotherapy significantly elevated event-free and overall survival times, $50 \%-70 \%$ of DLBCL patients can be cured, but nearly one-third of patients develop relapsed or refractory disease that remains a major cause of mortality and morbidity due to the limited therapeutic options[35]. The prognosis of patients with newly diagnosed DLBCL depends on the prognostic clinical and biological characteristics as defined in the previously developed International Prognostic Index (IPI)[6]. Because the patient prognosis within the IPI group is highly variable, novel biomarkers with potential implications for prognosis are of great importance[7]. The novel strategy for remedy seems to merit particular attention. Chemokines that binding to receptors play an essential role in tumorigenesis and pathological immune pathways and have been investigated as markers of adverse prognosis in nonHodgkin lymphomas[8, 9]. CCR2, a G protein-coupled receptor (GPCR) that combines with CCL2 mainly, can be detected in a diversity of cells, such as monocytes, memory T cells, as well as natural killer (NK) cells, dendritic cells and basophils[10-13]. The CCR2-CCL2 axis recruits inflammatory monocytes to the tumor microenvironment (TME) and promotes polarization of selectively activated M2-phenotypes, thereby contributing to immunosuppression and enhancing tumor vascularization, cancer extravasation 
and metastasis, including prostate cancer, pancreatic cancer, hepatocellular carcinoma[13-17]. This receptor and its ligands have been described in the tumor-promoting effects of malignant $B$ cells in hematologic neoplasms such as follicular lymphoma[18]. However, there are no data on its effect on DLBCL or its association as a prognostic marker for this condition. In this study, we evaluated the prognostic significance of this receptor in patients with DLBCL, and sought to investigate the role of alteration of CCR2 expression in DLBCL by in vitro and in vivo experiments.

\section{Materials And Methods}

\section{Patients and tissue samples}

We recruited 138 patients with DLBCL who performed pathological biopsies at the First Affiliated Hospital and the Second Affiliated Hospital of Anhui Medical University in the period 2004-2015, based on the WHO criteria[1] and all patients have been given informed consent. The following criteria: DLBCL pathologically substantiated; no preceding history of malignancy, transplantation, or immunosuppression; and laboratory data and follow-up information were available, were met by all patients. All cases were evaluated for CCR2 expression by utilizing immunohistological analysis and sections were independently reviewed by two highly experienced pathologists. Some $73 \%$ of all patients were treated with the $\mathrm{CHOP}$ (cyclophosphamide, hydroxydaunorubicin, vincristine and prednisone) and $27 \%$ with the CHOP regimen plus rituximab as primary therapy and follow-up at each of these two hospitals. This study was approved by the Institutional Review Board of Anhui Medical University and was conducted in accordance with The Code of Ethics of the World Medical Association (Declaration of Helsinki).

\section{Immunohistochemical staining}

Specimens from 138 patients, subcutaneous tumors and seeded organs were $4 \%$ formalin-fixed paraffinembedded. We used $4 \mu \mathrm{m}$ paraffin sections and the monoclonal antibodies of CCR2 (1:300 dilution, Abcam, USA) for patients specimens and anti-human CD20 (ZSGB-Bio, China), CCR2 (1:50 dilution, Bioss, China) for subcutaneous tumors and seeded organs in the study. The sections were dewaxed in xylene, stripped of xylene in a gradient of ethanol to reduce water. Antigens were then repaired using sodium citrate solution $(\mathrm{pH}=6)$ by high pressure antigen repair method. After using $3 \%$ hydrogen peroxide to block endogenous peroxidase and goat serum to reduce non-specific background staining, anti-CD20 and CCR2 antibodies were incubated on the sections overnight at $4^{\circ} \mathrm{C}$. After recovery to room temperature, biotin-labelled secondary antibodies (anti-rabbit) were added dropwise for 30 minutes at $37^{\circ} \mathrm{C}$, followed by room temperature action using horseradish peroxidase-labelled ovalbumin for 15 minutes. Finally, DAB was used for colour development followed by staining using hematoxylin. The sections of subcutaneous tumors and disseminated organs were also stained for hematoxylin and eosin. Stained sections were interpreted by two professional pathologists, with different magnifications ( $\times 200$ and 400) recorded in the same field of view for each section through an Olympus BX51TF microscope (Olympus, Japan). 


\section{Cell Culture}

SUDHL-4, SUDHL-6 and OCI-Ly8 (germinal center subtype, GCB) and SUDHL-2, OCI-Ly10 (activated subtype, ABC) are human-derived DLBCL cell lines. SUDHL-2, SUDHL-4 and OCI-Ly10 cell lines were generously gifted by Dr. Ding (The First Affiliated Hospital Of USTC China), SUDHL-6 and OCI-Ly8 cell lines were presented by Prof. Zhai Zhimin (The Second Hospital Of Anhui Medical University, China). Cell culture media consisted of 1640 (Hyclone, USA) plus 10\% fetal bovine serum (Gibco, USA) and 1\% penicillin/streptomycin (Beyotime, China). All cells were incubated at $37^{\circ} \mathrm{C}$ in a $5 \% \mathrm{CO} 2$ incubator.

\section{Proliferation assays}

Cell proliferation was measured with the use of Cell Counting Kit-8 (Beyotime, China). Briefly, SUDHL-2 and OCI-Ly 8 cells were resuspended and then inoculated $3 \times 104$ cells/100 $\mu$ L RPMI 1640 with $10 \%$ FBS

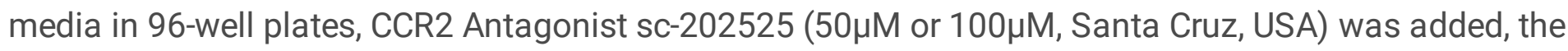
control group were treated with equal amounts of DMSO. The cells were incubated at $37^{\circ} \mathrm{C}$ and $5 \% \mathrm{CO} 2$ for 24,48 , and 72 hours, then $10 \mu \mathrm{L}$ of CCK8 solution was added. After incubation at $37^{\circ} \mathrm{C}$ for 2 hours, the absorbance of each well at $450 \mathrm{~nm}$ was detected. Results represent the average absorbance of five wells \pm SD.

\section{Migration Assay}

Migration experiments were performed using 8- $\mu \mathrm{m}$ pore size Transwell chambers (Corning Costar, USA), $2.5 \times 105$ cells in the upper chamber were resuspended in $100 \mu \mathrm{L}$ serum-free medium with or without CCR2 Antagonist $(100 \mu \mathrm{M})$ incubated for 2 hours at $37^{\circ} \mathrm{C}$, and $600 \mu \mathrm{L}$ serum-free medium in the lower chamber with or without rhMCP-1 (100ng/ml, R\&D System, USA), the amount of cells migrating to the lower chamber within 24 hours was counted by Cell Counting Machine (JIMBIO-FIL, China). Data represent the total number of cells that migrated to the lower chamber \pm SD.

\section{Apoptosis Analysis}

To estimate whether cells exposed to CCR2 Antagonist are undergoing apoptosis, We used Annexin VAPC Apoptosis Assay Kit (BestBio, China), following the manufacturer's instructions. In brief, SUDHL-2 cells and OCI-Ly8 cells were cultured in RPMI 1640 containing 10\% FBS with the addition of CCR2 Antagonist $(50 \mu \mathrm{M}$ or $100 \mu \mathrm{M})$ for 48 hours, then cells were washed twice with PBS and incubated with $2 \mu \mathrm{L}$ Annexin V-APC and $2 \mu \mathrm{L}$ propidium iodide (PI) at $4^{\circ} \mathrm{C}$ protected from light for 0.5 hour and then probed by flow cytometry. The control group was processed with equal quantities of DMSO.

\section{Western Blot}

The protein was extracted by centrifugation at $4^{\circ} \mathrm{C}$ and $12000 \mathrm{rpm} / \mathrm{min}$ for $20 \mathrm{~min}$ after collection and addition of RIPA (Beyotime, China) + 1\% PMSF (Beyotime, China). The preparation of BCA assay (Beyotime, China) for protein quantification was followed by adding Loading Buffer (Beyotime, China) and boiling for $10 \mathrm{~min}$. Separation of $20 \mu \mathrm{g}$ proteins by SDS polyacrylamide gel electrophoresis (SDSPAGE) and transfer of proteins to PVDF (polyvinylidene difluoride) membranes (Millipore, USA). Following 
blocking of the membranes by $5 \%$ skim milk in Tris-saline buffer supplemented with $0.1 \%$ Tween-20 at room temperature for 1 hour, they were subsequently performed with primary antibodies at $4^{\circ} \mathrm{C}$ overnight. The primary antibodies used were as follows: CCR2 antibody (1:1000, Bioworld, USA), p-PI3K antibody, pAkt antibody, p-P38 antibody (1:1000, all from Cell Signaling Technology, USA). After washing 3 times with TBST, the membranes were hatched with HRP-conjugated secondary antibody (1:5000, ZSGB-Bio, China) for 1 hour at room temperature. The bands were displayed using ECL reagent (Pierce, USA) with chemiluminescence imaging instrument (Tanon5200, China), and Image $J$ analyzed and calculated the grayscale values. The expression level of the objective protein was normalized to the level of $\beta$-actin (1:2000, ZSGB-Bio, China).

\section{In vivo proliferation assay}

4-6 weeks BALB/C female nude mice (16-22g) were obtained from GemPharmatech Co., Ltd (Nanjing, China). Mice were housed in specifc-pathogen-free (SPF) room under controlled temperature and humidity at the School of Basic Medical Sciences, Anhui Medical University. After a week of acclimatization, $1.5 \times 10 \otimes \mathrm{OCl}$-Ly8 cells were resuspended in 200ul PBS and injected subcutaneously into the right axilla of the nude mice. When tumor volume reached about $100 \mathrm{~mm}^{3}$, the mice were randomly divided into two groups, the CCR2 antagonist group $(\mathrm{N}=5)$ and the control group $(\mathrm{N}=6)$. The CCR2 antagonist group was injected intraperitoneally with CCR2 antagonist at a dose of $10 \mathrm{mg} / \mathrm{kg}$ every other day, the control group was given the same dose of DMSO every other day for 3 weeks (the day of administration was recorded as day 0 ). Recorded the mice weight and tumor size every other day and the tumor volume $=($ length $\mathrm{x}$ width $\times$ width $) / 2$. When the tumor volume reached about $1500 \mathrm{~mm}^{3}$, mice were euthanised and tumors were removed. Tumor growth and mice mass change curve were plotted.

\section{In vivo migration assays and bioluminescent imaging}

4-6 weeks female NOD-SCID mice (16-20g) were purchased from GemPharmatech Co., Ltd (Nanjing, China). 1.5×10区 Luciferin-labeled OCI-Ly8 cells were resuspended in 200ul PBS and injected into mice via tail vein, followed by random division into CCR2 antagonist group and control group. The CCR2 antagonist group was administrated CCR2 antagonist $(20 \mathrm{mg} / \mathrm{kg})$ intraperitoneally every day, while the control group was given the same dose of DMSO intraperitoneally. We observed the status and weight of the mice daily. They were euthanized according to animal ethics when showing signs of metastasis such as straightened hair and significant weight loss. We monitored the dissemination of intravenouslyinjected OCl-Ly8 via capturing bioluminescence (BLI) every 6 day using the bioluminescent imaging system (LagoHTX, USA ). Mice were anesthetized with 3.5\% isoflurane in oxygen and BLI was captured 10 minutes after intraperitoneal injection of firefly D-luciferin ( $3 \mathrm{mg} / \mathrm{per}$ mouse). The subcutaneous tumors and in vivo disseminated organs were removed for HE staining and immunohistochemical analysis. The animal experiments were approved by the Animal Ethics Committee of Anhui Medical University.

\section{Statistical analysis}


All data values were calculated as the mean \pm SEM from three independent experiments. We used either the chi-square or Fisher's exact test to compare the two sets of categorical variables. Univariate and multivariate analyses for PFS and OS were conducted using the Cox proportional hazard model. Survival curves were constructed by the Kaplan-Meier method and log-rank tests were used to compare differences between groups. For the cell culture trials and in vivo experiments, we determined statistical significance by a two-tailed Student's t-test using GraphPad Prism (version 8.0.2). The statistical calculations were conducted using SPSS software (version 25). $P<0.05$ indicates a statistically significant result.

\section{Results}

\section{Immunohistochemical CCR2 intensity and its correspondence with clinicopathological features}

We performed an assessment of CCR2 expression in 138 biopsies of DLBCL patients. All the clinical data of the enrolled DLBCL patients are available in Table 1. A high expression of CCR2 was observed in 74\% of the subjects and is expressed in tumor cells in both the cytoplasm and the cell membrane (Fig. 1A,B). The frequency of CCR2 expression progressively increased with higher IPI scores (Table 1, $P=0.001$ ). Positive CCR2 expression in DLBCL patients was remarkably correlated with Ann Arbor stage and LDH, respectively (Table $1, P<0.001$, respectively).

\section{High CCR2 expression is a negative prognostic factor affecting overall survival and progression-free survival in DLBCL patients}

To examine whether CCR2 expression is an independent prognostic predictives of progression-free survival (PFS) and overall survival (OS), we performed univariate and multivariate COX regression analysis. By employing univariate COX analysis, as shown in Table 2 and Table 3, CCR2 expression was a statistically significant predictor of PFS $(P<0.001)$ and OS $(P<0.001)$. The same was true for LDH $(P=$ 0.047 and $P=0.018$, respectively). Meanwhile, ECOG-PS was an independent prognostic component for PFS $(P=0.047)$. Furthermore, multivariate COX analysis revealed that CCR2 expression and LDH remained significant prognostic factors for PFS (Table 2, $P<0.001$ and $P=0.006$, separately). Similarly, CCR2 expression and LDH were proved to be significant prognostic agents for OS (Table $3, P<0.001$ and $P=0.011$, respectively).

\section{Correlation between CCR2 expression and survival rate in patients with DLBCL}

Following first-line chemotherapy, the overall effectiveness rate including complete response (CR) and partial response (PR) was 63\% in our research. The significant effect of CCR2 expression on the overall 
response rate is shown in Table $4(P<0.001)$. Kaplan-Meier analysis indicated that patients with CCR2 positive expression had significantly poorer PFS and OS (Fig. 2, log-rank $P<0.001$, respectively).

\section{Association of CCR2 expression driven the proliferation and migration in DLBCL cells}

We evaluated CCR2 expression in DLBCL cell lines by Western Blotting and found that CCR2 was expressed in all cell lines although at different degrees of expression (Fig. 1C, D). To verify the effect of the expression level of CCR2 on the proliferation of DLBDL cells, SUDHL-2 and OCl-Ly8 cells were supplemented with CCR2 antagonist (sc-202525, $50 \mu \mathrm{M}$ or $100 \mu \mathrm{M}$ ) or DMSO (equal in volume to the CCR2 antagonist treatment). We found that sc-202525 significantly inhibited the proliferation rate of SUDHL-2 (Fig. 3A,P < 0.01) and OCl-Ly8 cells (Fig. 3B, P<0.01) in comparison with the DMSO group. Identification of the migratory impact of CCR2 expression, we evaluated the migration induced by MCP-1 in DLBCL cell lines, recombinant human ( $\mathrm{rh}$ MCP-1 $(100 \mathrm{ng} / \mathrm{ml})$ was added to the lower chamber to stimulate cell migration, OCl-Ly8 cell increased their migration capacity in the presence of MCP-1, there was however no difference in SUDHL-2 cells. To evaluate whether MCP-1-driven migration was dependent on CCR2 expression, we evaluated whether exposure to sc-202525, a CCR2 antagonist, repressed this procedure. We found that sc-202525 significantly inhibited migration of SUDHL-2 and OCl-Ly8 cell lines (Fig. 3C).

\section{CCR2 expression promotes DLBCL cells anti-apoptotic ability}

To ascertain whether CCR2 activated intracellular signals, CCR2 antagonist (sc-202525) was applied to SUDHL-2 and OCl-Ly8 cells for $48 \mathrm{~h}$ and then cell apoptosis was detected by flow analysis using an apoptosis reagent kit, the same amount of DMSO was substituted for the negative group. As the illustration demonstrates, apoptosis increased after sc-202525 action on the cells, and the apoptosis rates of SUDHL-2 (Fig. 4A, P < 0.01) and OCl-Ly8 cells (Fig. 4B, P < 0.01) were approximately 3.5 times higher and 7 times higher than those of the control group, respectively.

\section{CCR2 activates PI3K-Akt signaling pathway and inhibits P38 signaling pathway in DLBCL}

The publication of the literature reported that the expression of PI3K/Akt signaling pathway and p38MARK signaling pathway in prostate cancer and breast cancer correlated with CCR2 in terms of level[19, 20]. However, the role of these signaling pathways in DLBCL has not been previously reported. To gain closer insights into the molecular mechanism by which CCR2 facilitates DLBCL cell survival, we treated SUDHL-2 and OCI-Ly8 cells with sc-202525 for 48 h, then screened p-PI3K protein, p-Akt protein, pP38 protein, p-STAT3 protein,p-STAT5 protein, $p$-Src protein, p-P44/42 protein and p-NF-kB protein expression by western blot. We have observed that p-PI3K and p-Akt expression was dramatically depressed, whereas p-p38 protein was significantly upregulated in both strains of cells (Fig. 4C,D). The $\mathrm{PI3K} /$ Akt signaling pathway and p38MARK signaling is engaged in regulating cell proliferation, metabolism and motility[21-23]. We offer consideration of the CCR2 expression involved in the 
proliferation and migration of DLBCL cells by activating PI3K/Akt signaling pathway, and induced apoptosis through activation of p38MARK signaling pathway.

\section{CCR2 antagonist inhibits subcutaneous tumor growth by blocking CCR2}

Based on the results of in vitro experiments, we performed in vivo experiments to explore whether CCR2 antagonist could inhibit tumor growth by blocking CCR2 in vivo. We transplanted 1.5×10区 OCl-Ly 8 cells into the right axillary subcutis of nude mice. When the tumor was apparent, mice were randomly divided into two groups. The CCR2 antagonist group was administrated with CCR2 antagonist $(10 \mathrm{mg} / \mathrm{kg})$ and the control group was given the same dose of DMSO every other day. We recorded the mass of mice and subcutaneous tumor size every other day. The results showed the tumor size and tumor weight treated with CCR2 antagonist reduced significantly compared to the control group (Fig. 5A,B,C,E), while the mice body weight showed no significant difference between two groups (Fig. 5D). When the subcutaneous tumor volume reached about $1500 \mathrm{~mm}^{3}$, the mice were euthanized and the tumors were removed and weighed. Immunohistochemistry and HE staining were performed on the subcutaneous tumors of both groups, showing CCR2 expression was significantly decreased in the CCR2 antagonist-treated group, compared to the DMSO treated group (Fig. 5F). These results confirmed that CCR2 antagonist can inhibit the growth of the subcutaneous tumors by blocking CCR2.

\section{CCR2 antagonist significantly inhibits tumor dissemination}

We have shown that CCR2 antagonist inhibited tumor growth by blocking CCR2, so next we explored CCR2 antagonist whether could inhibit tumor dissemination in vivo. $1.5 \times 10 \otimes 0$ Cl-Ly8 cells were resuspended in $200 \mathrm{ul} \mathrm{PBS}$ and injected intravenously into NOD-SCID mice. The mice were then randomly divided into two groups, one group was administrated intraperitoneally with CCR2 antagonist $(20 \mathrm{mg} / \mathrm{kg})$ daily and control group was injected intraperitoneally with DMSO. Bioluminescent imaging was performed every 6 days to monitor the dissemination of tumors in the mice. On day 12, a significant difference in tumor dissemination began to appear between the two groups, with parenchymal organ dissemination in the control group. The difference in dissemination between the two groups became more pronounced gradually (Fig. 6A). Bioluminescent imaging were analysed by software to obtain the fluorescence intensity of the tumor-disseminated organs and it showed the intensity of the control group increased significantly, with significant difference between the two groups (Fig. 6B). We euthanized the mice when they showed straightened hair and significant weight loss. Then all the organs of control group mice were removed for hematoxylin and eosin staining and immunohistochemical staining. Finally, we found the bone marrow, lung and central nervous system of the control mice were seeded by tumor, which was consistent with the bioluminescent imaging (Fig. 6C). These results confirmed CCR2 antagonist could inhibit the dissemination of tumor in vivo.

\section{Discussion}


In the current study, first of all, we investigated the prognostic significance of CCR2 expression in DLBCL patients. We found that CCR2 was high-expressed in 74\% of the DLBCL patients and the frequency of CCR2 expression gradually increased with increasing IPI scores. In addition, through multivariate analysis, we found for the first time that CCR2 receptor was an independent prognostic marker that affecting DLBCL patients. Our findings are paralleled by previous studies in which CCR2 expression was described as a prognostic factor associated with poor prognosis in liver cancer, lung cancer, prostate cancer, colorectal cancer and renal cancer[13, 24-28]. Furthermore, Kaplan-Meier analysis showed that patients with positive CCR2 expression had markedly inferior PFS and OS, which is consistent with the observations of B. Ou, et al[27].

Since CCR2 expression is upregulated in the majority of DLBCL patients, we investigated the expression of CCR2 in DLBCL cell lines to explore the role of this receptor on DLBCL cells. The results in vitro studies showed that CCR2 expression was detected by protein level analysis in DLBCL cell lines, including SUDHL-2, SUDHL-4, OCI-Ly10, SUDHL-6 and OCI-LY8 cells. Several published studies have identified the expression of CCR2 in cancer cells, including prostate cancer[26], multiple myeloma[29], kidney cancer[28] and lung cancer cells[25]. To examine the immediate effects of CCR2 expression on DLBCL cell lines, in vitro experiment, we treated SUDHL-2 and OCI-Ly8 cells with pharmacological concentrations of CCR2 antagonist Sc-202525. The result indicates high level of CCR2 stimulates the proliferation, migration and anti-apoptotic ability of DLBCL cells. The present results are consistent with previous studies, in which the CCR2 expression facilitates neoplastic progression of colorectal and ovarian cancer[27, 30].

Meanwhile, in vivo experiments, the results showed that the tumor was significantly reduced in volume after treatment with CCR2 antagonist, and bioluminescence imaging indicated that CCR2 antagonists significantly inhibit DLBCL cells dissemination, these results are consistent with our in vitro observations. Due to such an important role of CCR2 in tumor growth, invasion, migration and survival, optimal use of CCR2 inhibition may be part of a potential targeted therapy. The results of the current study indicate that CCR2 antagonists reduce cell viability, block metastasis, improve chemotherapeutic efficacy and overcome macrophage-induced depression [17, 31, 32]. A potent, selective and orally administered antagonist of CCR2, designated INCB3344, in both human and murine, the contents of these details have been published previously[33]. In fact, a phase II clinical trial was performed using the anti-CCR2 monoclonal antibody MLN1202 in patients with bone metastasis from cancer [34]. Furthermore, The first clinical trials of CCR2 antagonist called PF-04136309 (Pfizer) for its therapeutic potential in pancreatic adenocarcinoma are currently proceeding[35]. In addition, Chemocentryx has recently commenced a Phase lb trial of a CCR2 antagonist (CCX872) for the treatment of patients with irresectable pancreatic cancer[36]. These clinical researches will provide the most desired information on the security and potential therapeutic effects of CCR2 signalling blockade in people suffering from cancers.

The PI3K/Akt signalling pathway and p38 MARK signaling pathway are critical for many physiological and pathological conditions, such as regulating cell survival, growth, proliferation, angiogenesis, transcription, translation and metabolism [20,37-39]. To further investigate the molecular mechanisms 
by which CCR2 expression promotes the survival of DLBCL cells, Western blot analysis for the PI3K/Akt signalling pathway and the P38 MARK signalling pathway showed that CCR2 antagonists significantly inhibited the activation of the PI3K/Akt signalling pathway, yet activated the P38 MARK signalling pathway in vitro. Similarly, the data according to Roca et al, the CCL2-CCR2 axis regulates autophagy and increases survivin expression via the PI3K/Akt pathway, providing a strong survival advantage in prostate cancer PC-3 cells[40]. In ovarian cancer, Chao et al. found that binding of large omental adipocytes to their cognate receptor CCR2 promotes tumor cell migration and omental metastasis through activation of the PI3K/Akt pathway[30]. In addition, Erwin $\mathrm{F}$ et al. found that apoptotic stimuli sometimes trigger the activation of p38 via secondary pathways, such as the production of reactive oxygen species (ROS)[20]. These results highly emphasized the carcinogenic role of CCR2 in DLBCL and that targeting CCR2 may be identified as a very promising therapeutic strategy for DLBCL.

In conclusion, we demonstrated that the CCR2 receptor is an independent prognostic marker that affects patients with DLBCL and is associated with poorer OS and PFS. Furthermore, we found that CCR2 antagonists inhibited DLBCL cell proliferation, migration and promotes apoptosis in vitro as well as in vivo by activating the PI3K/Akt signaling pathway and the p38 MARK signaling pathway. These discoveries suggest that targeting CCR2 may be a promising treatment option for DLBCL.

\section{Declarations}

\section{Ethics approval and consent to participate}

This study was approved by the Institutional Review Board of Anhui Medical University and was conducted in accordance with The Code of Ethics of the World Medical Association (Declaration of Helsinki).

\section{Consent for publication}

Not applicable.

\section{Availability of data and materials}

The datasets used and/or analysed during the current study are available from the corresponding author on reasonable request.

\section{Competing interests}

The authors declare that they have no competing interests.

\section{Funding}

This work was supported by National Natural Science Foundation of China (81700194), College Natural Science Foundation of Anhui Province (KJ2021 A0214). 


\section{Authorship contributions}

Yan-Li Li designed the research, collected the clinical data, supervised the experiments and revised the manuscript. Quan-Quan Hu analyzed the clinical data, performed the experiments in vitro, completed statistical analysis and wrote the paper. Zhao-Feng Wen and Qi-Tang Huang helped to perform in vivo experiments. Qian Li and Zhi-Min Zhai also supervised the experiments and revised the manuscript.

\section{Acknowledgements}

We greatly appreciate Dr. Zhi-Min Zhai and Dr. Shu-Dao Xiong of The Second Affiliated Hospital of Anhui Medical University for providing the experimental platform. We are also thankful to Dr. Ding from The First Affiliated Hospital Of USTC for providing the DLBCL cells. We also thank Associate Professor Yan-Li

Li for her support with statistical analysis. We also recognize the use of a Nanotoxicology platform for the bioluminescent imaging of the tumor-bearing mouse.

\section{Conflict of interest}

The authors declare no competing financial interests.

\section{References}

1. Swerdlow, S.H., et al., The 2016 revision of the World Health Organization classification of lymphoid neoplasms. Blood, 2016. 127(20): p. 2375-90.

2. Chihara, T., et al., Frequency of intravascular large B-cell lymphoma in Japan: study of the Osaka Lymphoma Study Group. J Hematol Oncol, 2011. 4: p. 14.

3. Feugier, P., et al., Long-term results of the R-CHOP study in the treatment of elderly patients with diffuse large B-cell lymphoma: a study by the Groupe d'Etude des Lymphomes de I'Adulte. J Clin Oncol, 2005. 23(18): p. 4117-26.

4. Camicia, R., H.C. Winkler, and P.O. Hassa, Novel drug targets for personalized precision medicine in relapsed/refractory diffuse large B-cell lymphoma: a comprehensive review. Mol Cancer, 2015. 14: p. 207.

5. Coiffier, B., et al., CHOP chemotherapy plus rituximab compared with $\mathrm{CHOP}$ alone in elderly patients with diffuse large-B-cell lymphoma. N Engl J Med, 2002. 346(4): p. 235-42.

6. Ghielmini, M., et al., ESMO Guidelines consensus conference on malignant lymphoma 2011 part 1: diffuse large B-cell lymphoma (DLBCL), follicular lymphoma (FL) and chronic lymphocytic leukemia (CLL). Ann Oncol, 2013. 24(3): p. 561-76.

7. Perry, A.M., Z. Mitrovic, and W.C. Chan, Biological prognostic markers in diffuse large B-cell lymphoma. Cancer Control, 2012. 19(3): p. 214-26.

8. Salven, P., et al., Simultaneous elevation in the serum concentrations of the angiogenic growth factors VEGF and bFGF is an independent predictor of poor prognosis in non-Hodgkin lymphoma: a single-institution study of 200 patients. Blood, 2000. 96(12): p. 3712-8. 
9. Nagarsheth, N., M.S. Wicha, and W. Zou, Chemokines in the cancer microenvironment and their relevance in cancer immunotherapy. Nat Rev Immunol, 2017. 17(9): p. 559-572.

10. Balkwill, F.R., The chemokine system and cancer. J Pathol, 2012. 226(2): p. 148-57.

11. Brummer, G., et al., CCR2 signaling in breast carcinoma cells promotes tumor growth and invasion by promoting CCL2 and suppressing CD154 effects on the angiogenic and immune microenvironments. Oncogene, 2020. 39(11): p. 2275-2289.

12. Brummer, G., et al., Chemokine Signaling Facilitates Early-Stage Breast Cancer Survival and Invasion through Fibroblast-Dependent Mechanisms. Mol Cancer Res, 2018. 16(2): p. 296-308.

13. Li, X., et al., Targeting of tumour-infiltrating macrophages via CCL2/CCR2 signalling as a therapeutic strategy against hepatocellular carcinoma. Gut, 2017. 66(1): p. 157-167.

14. Qian, B.Z., et al., CCL2 recruits inflammatory monocytes to facilitate breast-tumour metastasis. Nature, 2011. 475(7355): p. 222-5.

15. Yao, W., et al., A Natural CCR2 Antagonist Relieves Tumor-associated Macrophage-mediated Immunosuppression to Produce a Therapeutic Effect for Liver Cancer. EBioMedicine, 2017. 22: p. 5867.

16. Ding, X., et al., TR4 nuclear receptor promotes prostate cancer metastasis via upregulation of CCL2/CCR2 signaling. Int J Cancer, 2015. 136(4): p. 955-64.

17. Sanford, D.E., et al., Inflammatory monocyte mobilization decreases patient survival in pancreatic cancer: a role for targeting the CCL2/CCR2 axis. Clin Cancer Res, 2013. 19(13): p. 3404-15.

18. Husson, H., et al., MCP-1 modulates chemotaxis by follicular lymphoma cells. Br J Haematol, 2001. 115(3): p. 554-62.

19. Zhang, J., Y. Lu, and K.J. Pienta, Multiple roles of chemokine (C-C motif) ligand 2 in promoting prostate cancer growth. J Natl Cancer Inst, 2010. 102(8): p. 522-8.

20. Wagner, E.F. and A.R. Nebreda, Signal integration by JNK and p38 MAPK pathways in cancer development. Nat Rev Cancer, 2009. 9(8): p. 537-49.

21. Alzahrani, A.S., PI3K/Akt/mTOR inhibitors in cancer: At the bench and bedside. Semin Cancer Biol, 2019. 59: p. 125-132.

22. Tewari, D., et al., Natural products targeting the PI3K-Akt-mTOR signaling pathway in cancer: A novel therapeutic strategy. Semin Cancer Biol, 2019.

23. Li, M.Q., et al., Chemokine CCL2 enhances survival and invasiveness of endometrial stromal cells in an autocrine manner by activating Akt and MAPK/Erk1/2 signal pathway. Fertil Steril, 2012. 97(4): p. 919-29.

24. Korbecki, J., et al., CC Chemokines in a Tumor: A Review of Pro-Cancer and Anti-Cancer Properties of the Ligands of Receptors CCR1, CCR2, CCR3, and CCR4. Int J Mol Sci, 2020. 21(21).

25. Wei, J., D. Fang, and W. Zhou, CCR2 and PTPRC are regulators of tumor microenvironment and potential prognostic biomarkers of lung adenocarcinoma. Ann Transl Med, 2021. 9(18): p. 1419. 
26. Lu, Y., et al., CCR2 expression correlates with prostate cancer progression. J Cell Biochem, 2007. 101(3): p. 676-85.

27. Ou, B., et al., A positive feedback loop of $\beta$-catenin/CCR2 axis promotes regorafenib resistance in colorectal cancer. Cell Death Dis, 2019. 10(9): p. 643.

28. Wyler, L., et al., Brain metastasis in renal cancer patients: metastatic pattern, tumour-associated macrophages and chemokine/chemoreceptor expression. Br J Cancer, 2014. 110(3): p. 686-94.

29. Vande Broek, I., et al., Clinical significance of chemokine receptor (CCR1, CCR2 and CXCR4) expression in human myeloma cells: the association with disease activity and survival. Haematologica, 2006. 91(2): p. 200-6.

30. Sun, C., et al., MCP-1/CCR-2 axis in adipocytes and cancer cell respectively facilitates ovarian cancer peritoneal metastasis. Oncogene, 2020. 39(8): p. 1681-1695.

31. Mitchem, J.B., et al., Targeting tumor-infiltrating macrophages decreases tumor-initiating cells, relieves immunosuppression, and improves chemotherapeutic responses. Cancer Res, 2013. 73(3): p. 1128-41.

32. Maxwell, P.J., et al., Tumor-derived CXCL8 signaling augments stroma-derived CCL2-promoted proliferation and CXCL12-mediated invasion of PTEN-deficient prostate cancer cells. Oncotarget, 2014. 5(13): p. 4895-908.

33. Xue, C.B., et al., Discovery of INCB3344, a potent, selective and orally bioavailable antagonist of human and murine CCR2. Bioorg Med Chem Lett, 2010. 20(24): p. 7473-8.

34. Kenneth J. P., et al., S0916, MLN1202 in Treating Patients With Bone Metastases. ClinicalTrials. gov NCT01015560(2015)

35. Andrea W.G., et al., FOLFIRINOX Plus PF-04136309 in Patients With Borderline Resectable and Locally Advanced Pancreatic Adenocarcinoma. ClinicalTrials. gov NCT01413022.

36. Phase 1b Study of CCX872-B in Patients With Pancreatic Adenocarcinoma. ClinicalTrials. gov NCT02345408

37. Fresno Vara, J.A., et al., PI3K/Akt signalling pathway and cancer. Cancer Treat Rev, 2004. 30(2): p. 193-204.

38. Nepstad, I., et al., The PI3K-Akt-mTOR Signaling Pathway in Human Acute Myeloid Leukemia (AML) Cells. Int J Mol Sci, 2020. 21(8).

39. Xia, P. and X.Y. Xu, PIJK/Akt/mTOR signaling pathway in cancer stem cells: from basic research to clinical application. Am J Cancer Res, 2015. 5(5): p. 1602-9.

40. Roca, H., Z. Varsos, and K.J. Pienta, CCL2 protects prostate cancer PC3 cells from autophagic death via phosphatidylinositol 3-kinase/AKT-dependent survivin up-regulation. J Biol Chem, 2008. 283(36): p. 25057-73.

\section{Tables}

Table 1. Association between CCR2 expression and patients' clinico-pathological features 


\begin{tabular}{|c|c|c|c|c|c|}
\hline \multirow[t]{2}{*}{ Clinico-pathological features } & \multicolumn{2}{|c|}{ patients } & \multicolumn{3}{|c|}{ CCR2 expression } \\
\hline & & $\%$ & Negative & Positive & $P$ \\
\hline \multicolumn{6}{|l|}{ Gender } \\
\hline Male & 73 & 53 & $17(12)$ & $56(41)$ & 0.427 \\
\hline Female & 65 & 47 & $19(14)$ & $46(33)$ & \\
\hline \multicolumn{6}{|l|}{ Age } \\
\hline Median (range) & \multicolumn{2}{|c|}{$58(13-84)$} & & & \\
\hline$<60$ & 80 & 58 & $24(17)$ & $56(41)$ & 0.219 \\
\hline$\geq 60$ & 58 & 42 & $12(9)$ & $46(33)$ & \\
\hline \multicolumn{6}{|l|}{ Ann Arbor stage } \\
\hline I/II & 67 & 49 & $27(20)$ & $40(29)$ & $<0.001$ \\
\hline III/IV & 71 & 51 & $9(6)$ & $62(45)$ & \\
\hline \multicolumn{6}{|l|}{ Extranodal sites of disease } \\
\hline$\leq 1$ & 101 & 73 & $30(22)$ & $71(51)$ & 0.110 \\
\hline$>1$ & 37 & 27 & $6(4)$ & $31(23)$ & \\
\hline \multicolumn{6}{|l|}{ ECOG-PS } \\
\hline$\leq 1$ & 79 & 57 & $25(18)$ & $54(39)$ & 0.085 \\
\hline$>1$ & 59 & 43 & $11(8)$ & $48(35)$ & \\
\hline \multicolumn{6}{|l|}{ LDH } \\
\hline$<$ normal & 91 & 66 & $33(24)$ & $58(42)$ & $<0.001$ \\
\hline$\geq$ normal & 47 & 34 & $3(2)$ & $44(32)$ & \\
\hline \multicolumn{6}{|l|}{ IPI } \\
\hline $0-2$ & 84 & 61 & $30(22)$ & $53(39)$ & 0.001 \\
\hline $3-5$ & 54 & 39 & $6(4)$ & $48(35)$ & \\
\hline \multicolumn{6}{|l|}{ Chemotherapy } \\
\hline CHOP & 101 & 73 & $24(17)$ & $77(56)$ & 0.304 \\
\hline R-CHOP & 37 & 27 & $12(9)$ & $25(18)$ & \\
\hline \multicolumn{6}{|l|}{ Subtype } \\
\hline $\mathrm{ABC}$ & 79 & 57 & $19(14)$ & $60(44)$ & 0.528 \\
\hline GCB & 59 & 43 & $17(12)$ & $42(30)$ & \\
\hline
\end{tabular}

Table 2. Univariate and multivariate COX regression analysis for progression-free survival in DLBCL patients. 
Progression-free survival

Variables

Univariate COX Regression Multivariate COX Regression

$\begin{array}{lll}\text { HR (IC 95\%) } & P & \text { HR (IC 95\%) }\end{array}$

\begin{tabular}{llccc}
\hline CCR2 (+/-) & $14.527(6.692-31.536)$ & $<0.001$ & $11.002(5.285-22.903)$ & $<0.001$ \\
LDH (Norma1/High) & $1.969(1.008-3.848)$ & 0.047 & $2.337(1.274-4.289)$ & 0.006 \\
ECOG-PS $(\leqslant 1 />1)$ & $0.561(0.317-0.993)$ & 0.047 & $0.642(0.395-1.044)$ & 0.074 \\
IPI $(0-2 / 3-5)$ & $1.063(0.356-3.175)$ & 0.913 & & \\
Ann Arbor stage & $0.687(0.351-1.345)$ & 0.273 & \\
(I-IIIII-IV) & $1.993(0.788-5.045)$ & 0.145 & & \\
$\begin{array}{l}\text { Extranodal sites of disease } \\
\text { ( } 1 />1)\end{array}$ & $0.625(0.387-1.012)$ & 0.056 & & \\
GCB (yes $/$ no $)$ & $1.270(0.770-2.096)$ & 0.349 & & \\
Age $(<60 / \geqslant 60)$ & & &
\end{tabular}

Table 3. Univariate and multivariate COX regression analysis for overall survival in DLBCL patients.

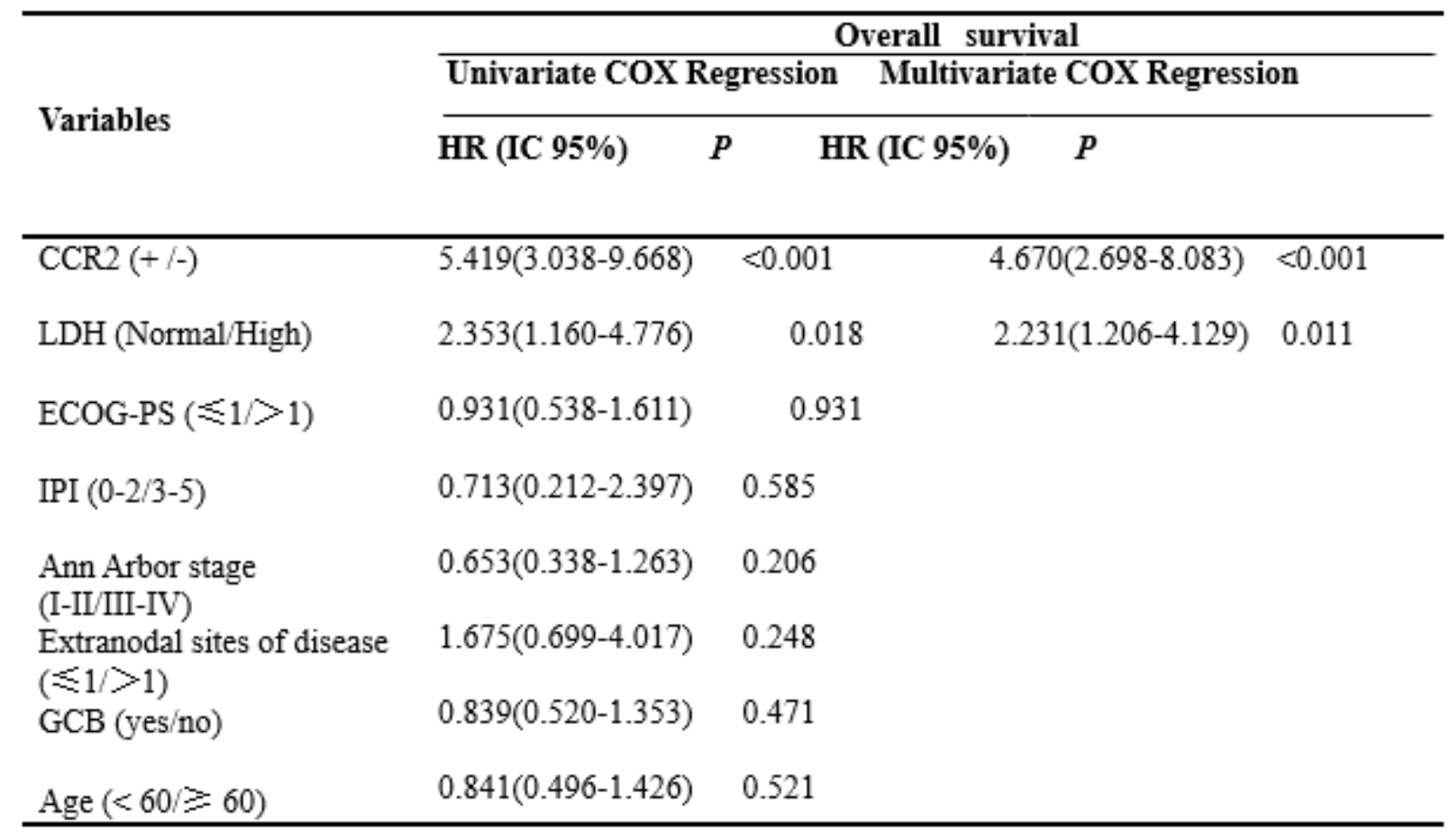

Table 4. Response rate according to CCR2 expression 


\begin{tabular}{lcccccc}
\hline Characteristic & \multicolumn{2}{c}{ Response } & & & \multicolumn{2}{c}{ Response rate } \\
\cline { 2 - 3 } \cline { 5 - 6 } & CR/PR & PD & & & \\
\hline CCR2 & 32 & 4 & & & \\
Negative & 5 & 47 & & & 89 & $<0.001$ \\
Positive & & & & 54 & $<0.001$ \\
\hline
\end{tabular}

Figures
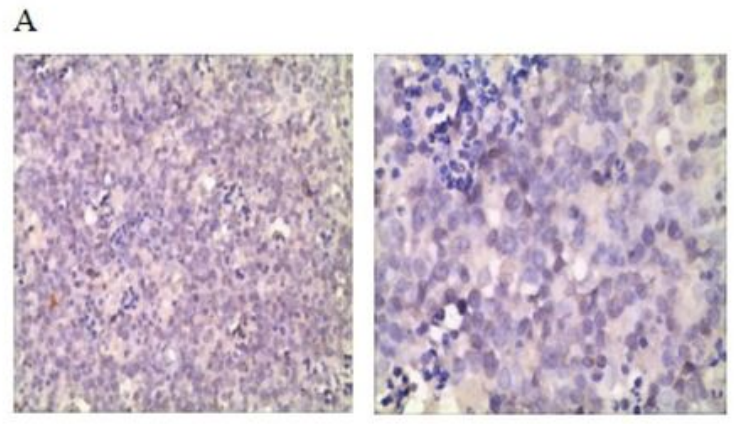

B

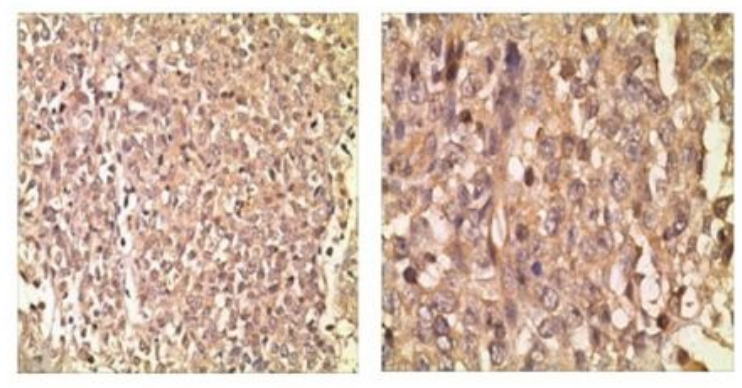

C
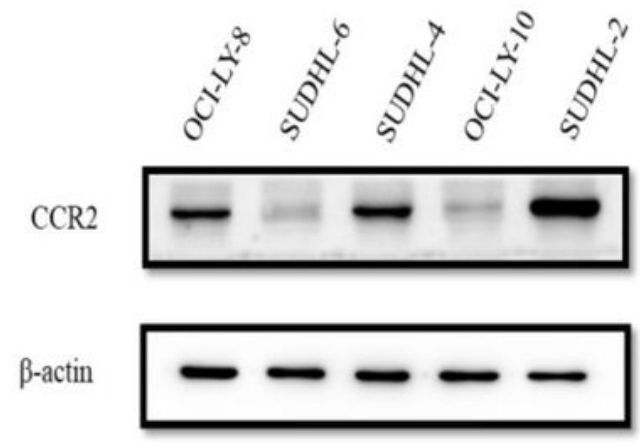

D

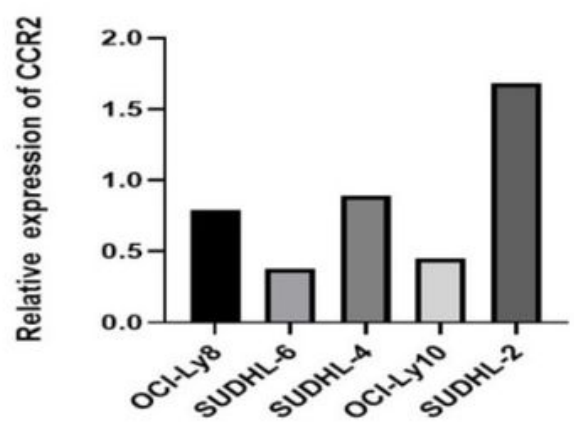


Figure 1

\section{Expression of CCR2 in DLBCL tissues and cell lines .}

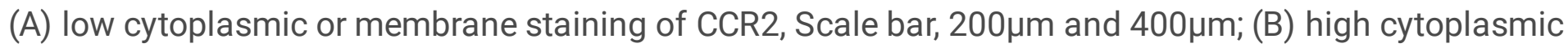
or membrane staining of CCR2, Scale bar, $200 \mu \mathrm{m}$ and $400 \mu \mathrm{m}$; (C) CCR2 were expressed in human DLBCL cell lines of SUDHL-2, SUDHL-4, SUDHL-6, OCI-Ly8 and OCI-Ly10; (D) Relative CCR2 protein expression was higher in SUDHL-2, SUDHL-4 and OCI-Ly8 than in SUDHL-6 or OCI-Ly10 cells.

A

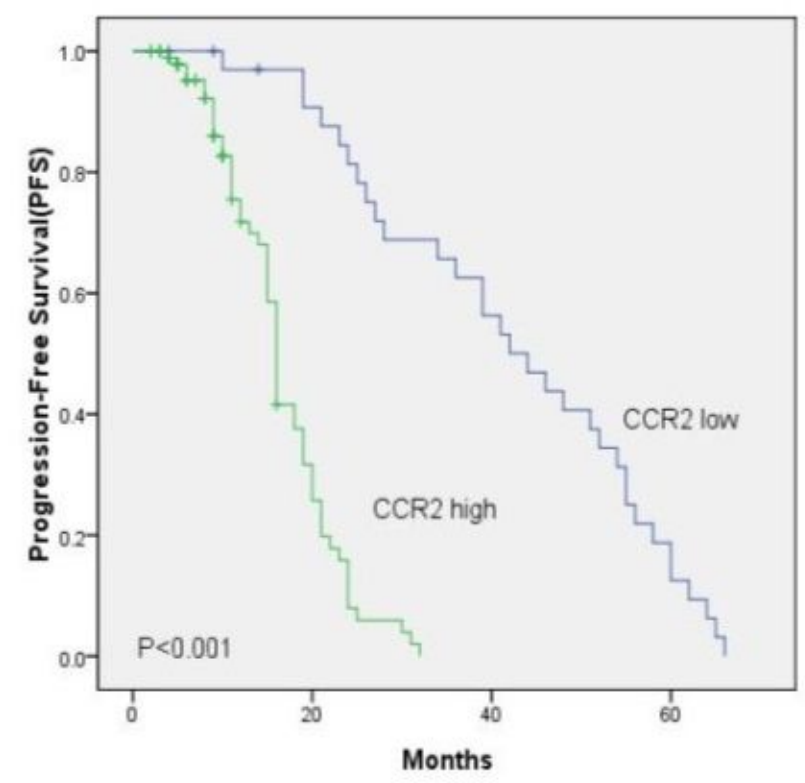

B

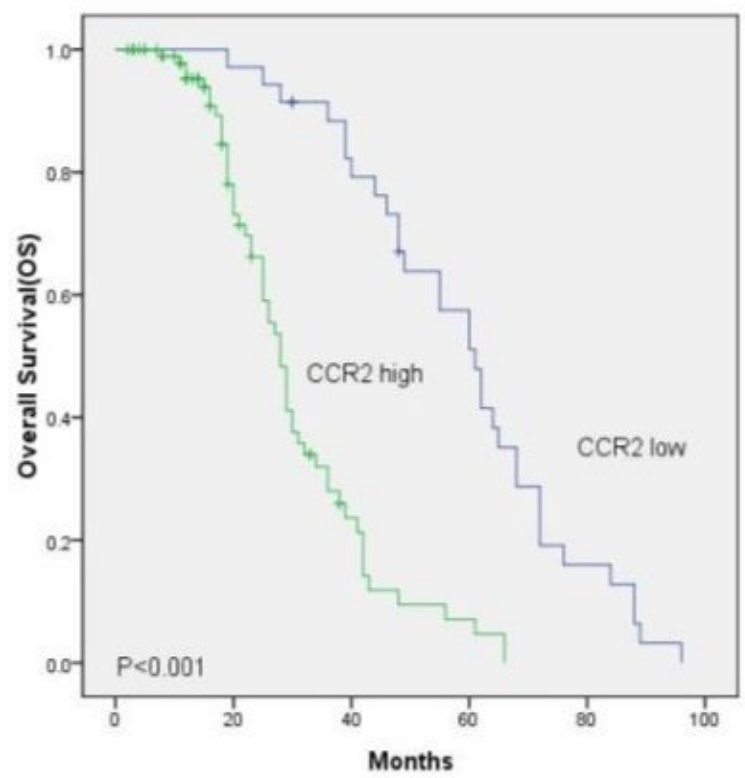

Figure 2

Kaplan-Meier analysis of PFS and OS according to the expression of CCR2 in patients with DLBCL.

(A) Patients expressing CCR2 in tumor cell (CCR2 high) showed a decreased PFS and (B) a reduced OS than patients who did not express this receptor (CCR2 low). $P$ values were calculated using the Log rank test. 
A

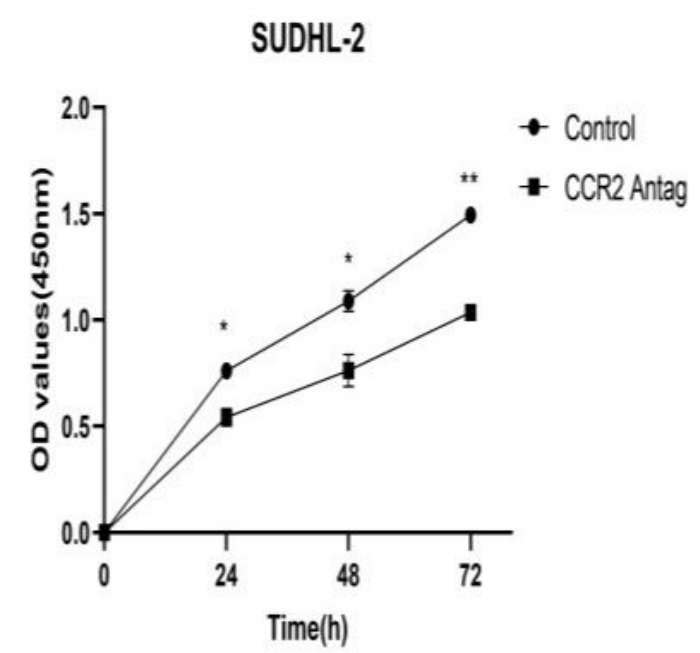

B

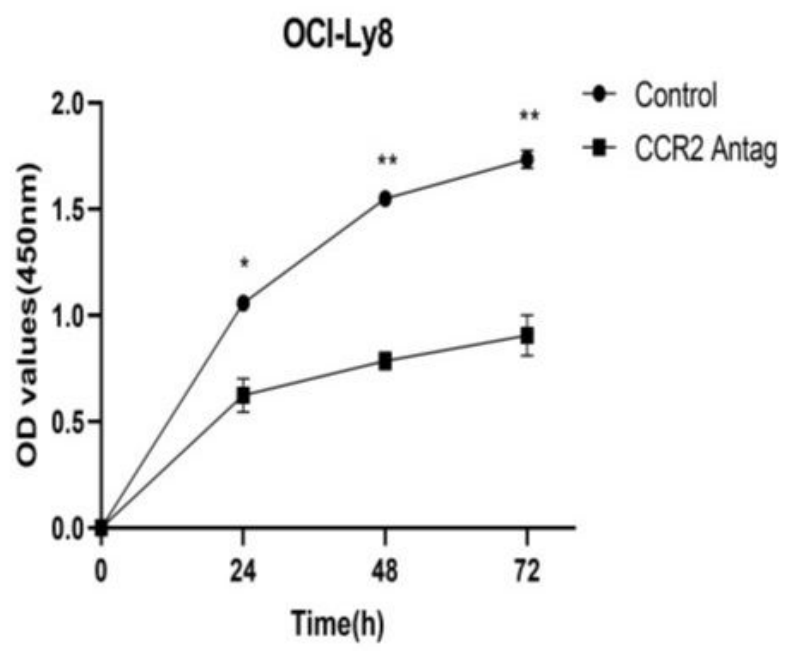

$\mathrm{C}$

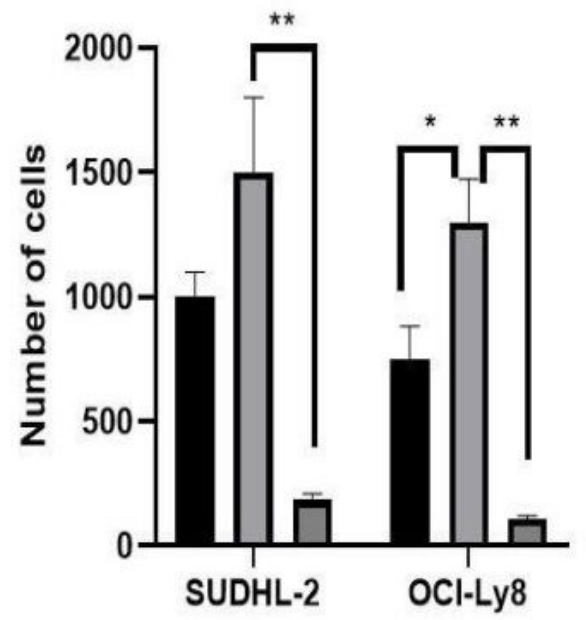

- Control

ㅁ.1

口 MCP-1+CCR2 Antagonist

\section{Figure 3}

CCR2 expression driven the proliferation and migration in DLBCL cell lines.

(A) SUDHL-2 cells. (B) OCI-Ly8 cells. To determine growth, SUDHL-2 or OCl-Ly8 cells ( $2 \times 10^{4} /$ well) were seeded into a 96-well plate and cultured for $24-72 \mathrm{~h}$ with RPMI 1640 and $10 \%$ fetal bovine serum and relative cell numbers in each well measured by CCK8. Cells were grown in the presence of CCR2 antagnoist sc-202525. The control cells were treated with equivalent amounts of DMSO. (C) Association of CCR2 expression with MCP-1 driven migration in DLBCL cell lines. To determine migration,cells were incubated in serum-free media in the presence or absence of CCR2 antagonist sc-202525 $(100 \mu \mathrm{M})$ prior 
to and during the transwell migration assay. Recombinant human (rh) MCP-1 (100ng/ml) was added to the lower chamber to stimulate cell migration. Cells unexposed to rhMCP-1 were used as control. OCl-Ly8 cell increased their migration capacity in the presence of MCP-1, however there was no difference in SUDHL-2 cells. We found that sc-202525 significantly inhibited migration spurred by MCP-1 in SUDHL-2 and OCI-Ly 8 cell lines. Data are presented as the mean \pm SEM $(n=3) .{ }^{*}$ value $<0.05,{ }^{*} P$ value $<0.01$.

A
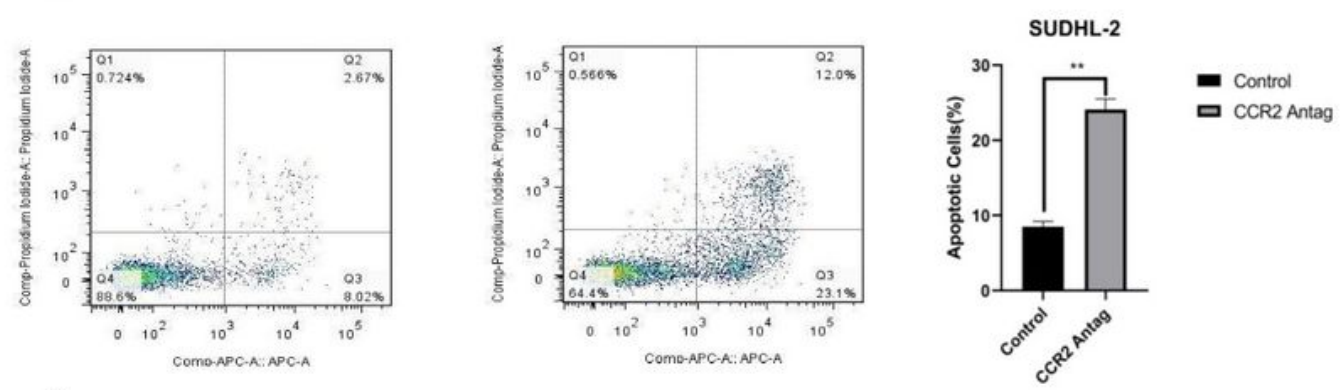

B
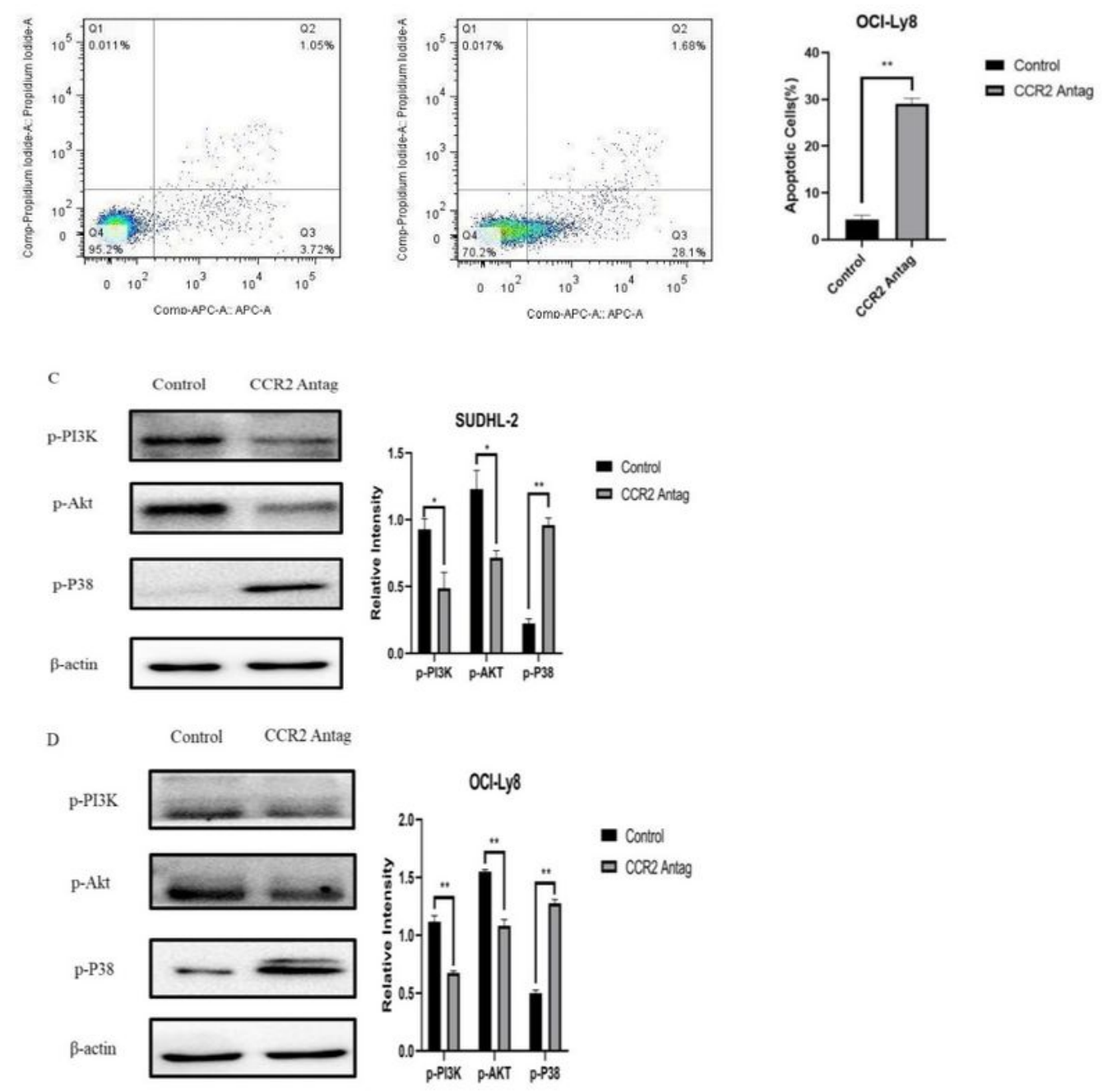

Figure 4 
CCR2 expression promotes anti-apoptotic ability and signalling pathways associated with biological behaviour in DLBCL.

To determine apoptotic, SUDHL-2 cells (A) and OCI-Ly8 cells (B) either treated with DMSO (Control) or after treatment with $50 \mu \mathrm{M} \mathrm{sc}-202525$ for $48 \mathrm{~h}$ and cell viability was measured by FACS used Annexin V APC-PI Apoptosis kit. To obtain a deeper understanding of the molecular mechanisms of CCR2 on DLBCL cells, we treated SUDHL-2 cells (C) and OCI-Ly8 cells (D) with sc-202525 for $48 \mathrm{~h}$, detection p-PI3K protein, p-Akt protein, p-P38 protein, p-STAT3 protein,p-STAT 5 protein, $\mathrm{p}$-Src protein, p-P44/42 protein and p-NF-kB protein expression by Western Blotting. Cells dealed with CCR2 antagonist p-PI3K and p-Akt expression decreased, however $\mathrm{p}-\mathrm{P} 38$ protein express raised vs. the control cells (treated with DMSO). Data are presented as the mean \pm SEM . ${ }^{P}$ value $<0.05, * * P$ value $<0.01$.

A
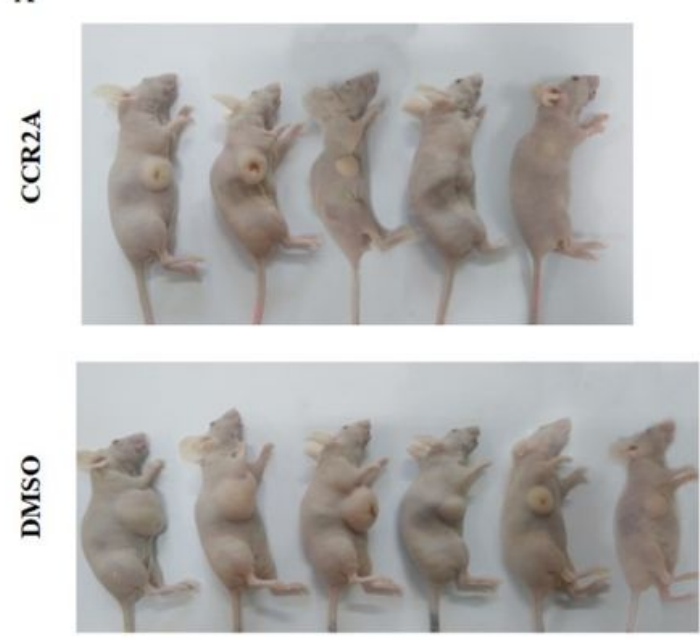

B

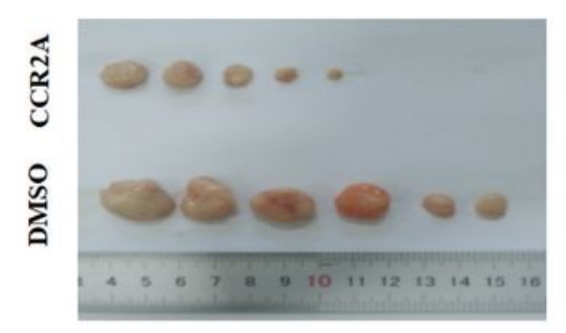

C

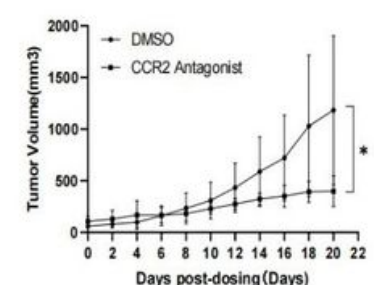

E

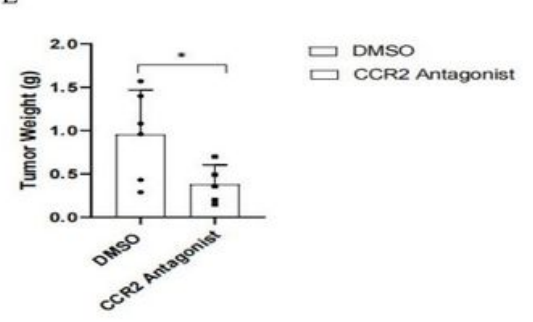

D

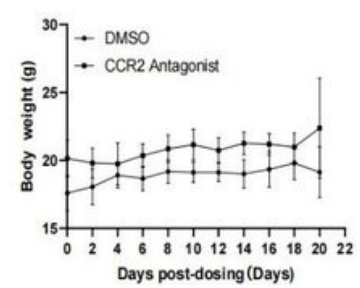

Days post-dosing (Days)

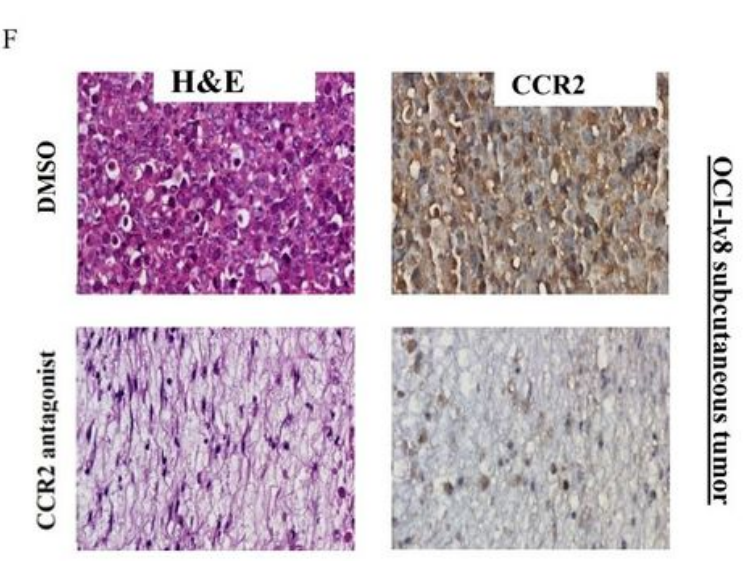

Figure 5

CCR2 antagonist inhibits the growth of subcutaneous tumor. $1.5 \times 10 \otimes \mathrm{OCl}-\mathrm{ly} 8$ cells were transplanted into the right sides of nude mice. When tumor volume reached about $100 \mathrm{~mm}^{3}$, we divided the mice into two 
groups, which administrated CCR2 antagonist $(10 \mathrm{mg} / \mathrm{kg})$ or DMSO intraperitoneally every other day. (A) Representative images of subcutaneous tumours treated with CCR2 antagonist or DMSO. (B) Subcutaneous tumours were removed from euthanized mice. (C) The tumor volumes of two groups ( means $\pm S E M ; C C R 2$ antagonist, $\left.n=5 ; D M S O, n=6 ;{ }^{*} p<0.05\right)$. (D) The mice weight of two groups (means $\pm S E M$ ). (E) The subcutaneous tumor weights of two groups (means $\pm S E M$, $\left.{ }^{*} p<0.05\right)$. (F) H\&E staining and immunohistochemistry (CCR2) were performed on OCl-ly8 subcutaneous tumors after CCR2 antagonist treatment. Scale bar, $62 \mu \mathrm{m}$; original magnification $\times 200$. H\&E, hematoxylin and eosin staining.

A
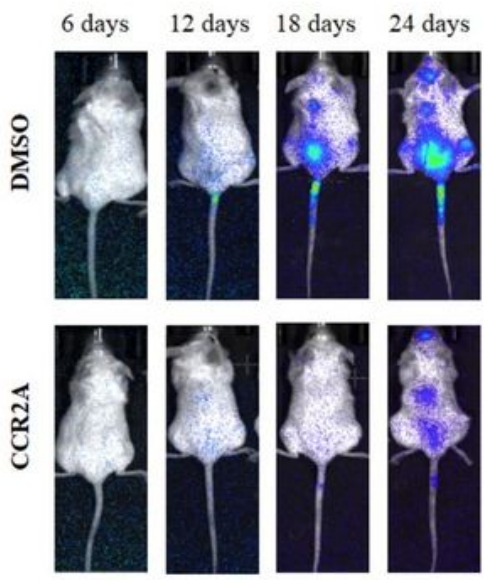

B

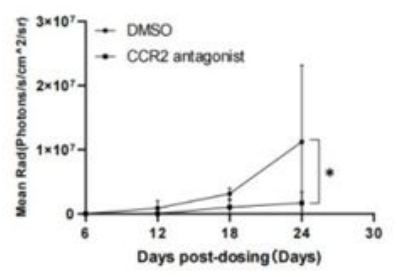

$\mathrm{C}$

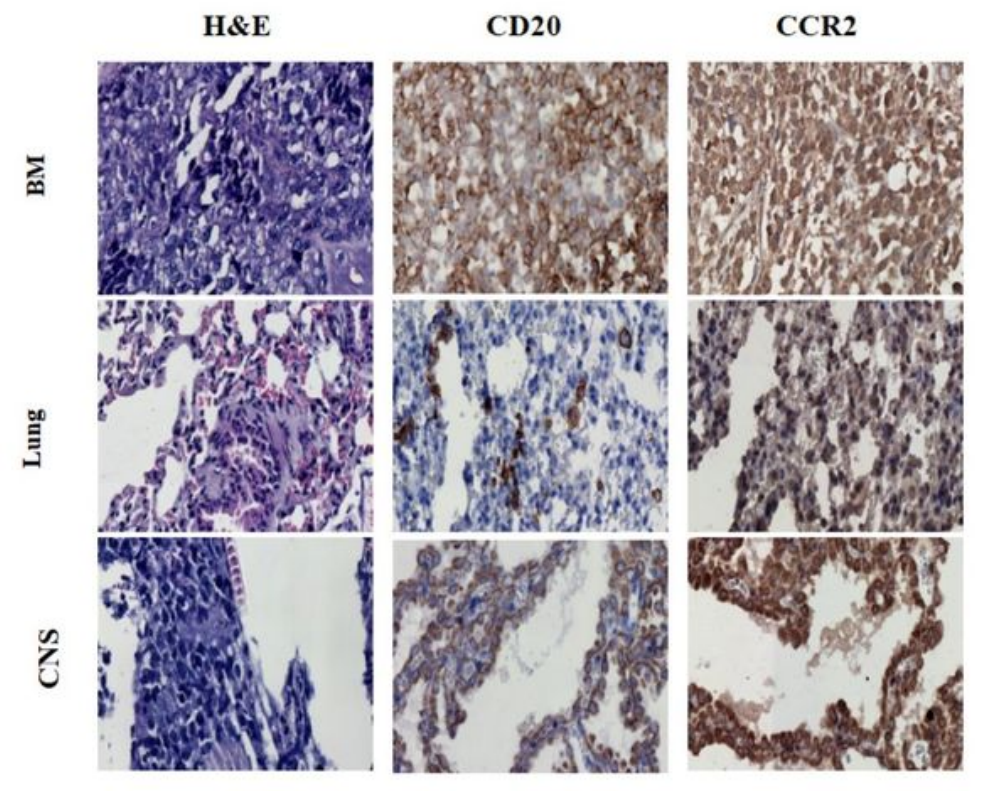




\section{Figure 6}

\section{CCR2 antagonist inhibits the dissemination of tuomr.}

$1.5 \times 10 \otimes \mathrm{OCl}-\mathrm{ly} 8$ cells were injected intravenously into NOD-SCID mice, which were intraperitoneally dosed daily with CCR2 antagonist $(20 \mathrm{mg} / \mathrm{kg})$ or DMSO. The tumor dissemination was monitored through bioluminescent imaging (BLI) every six days. After 24 days, the mice were euthanized and removed all organs for H\&E and immunohistochemistry. Finally, we found the BM, lungs and CNS were disseminated organs with being consistent with the BLI. (A) The BLI of CCR2 antagonist or DMSO treated mice. (B) The BLI curve of CCR2 antagonist or DMSO treated mice ( means $\pm S E M,{ }^{*} p<0.05$ ). (C) The expression of CD20 and CCR2 of BM, lungs and CNS of the disseminated mice. Scale bar, $62 \mu \mathrm{m}$; original magnification $\times 200$. $\mathrm{H} \& \mathrm{E}$, hematoxylin and eosin staining; BM, bone marrow; CNS, central nervous system. 